\title{
Effects of resonant phonon scattering from internal molecular modes on the thermal conductivity of molecular glasses
}

\author{
A. I. Krivchikov, A. N. Yushchenko, and O. A. Korolyuk \\ B. Verkin Institute for Low Temperature Physics and Engineering of NAS Ukraine, Kharkov 61103, Ukraine \\ F. J. Bermejo, R. Fernandez-Perea, and I. Bustinduy* \\ Instituto de Estructura de la Materia, CSIC, and Departamento Electricidad y Electrónica-Unidad Asociada CSIC, Facultad de Ciencia \\ y Tecnología, Universidad del País Vasco/EHU, P.O. Box 644, E-48080 Bilbao, Spain \\ M. A. González \\ Institute Laue Langevin, 6 Rue Jules Horowitz, F-38042-Grenoble Cedex 9, France \\ (Received 17 August 2007; revised manuscript received 22 October 2007; published 10 January 2008)
}

\begin{abstract}
The thermal conductivity $\kappa(T)$ of the crystalline and glassy phases of the two isomers of propyl alcohol has been measured. The two isomers differ by a minor chemical detail involving the position of the hydroxyl group with respect to the carbon backbone. Such a difference in molecular structure leads, however, to disparate behaviors for the temperature dependence of $\kappa(T)$, for both glass and crystal states. The $\kappa(T)$ for the glass shows for 1-propanol an anomalously large plateau region comprising temperatures within 6-90 K, while data for isomeric 2-propanol show only a small plateau up to $10 \mathrm{~K}$ which is comparable to data on lower alcohols. The results emphasize the role played by internal molecular degrees of freedom as sources of strong resonant phonon scattering.
\end{abstract}

DOI: 10.1103/PhysRevB.77.024202

PACS number(s): 61.43.Fs, 65.60.+a, 66.10.C-, 63.50.-x

\section{INTRODUCTION}

Significant progress has been registered in the past decades concerning the dynamics of disordered matter, particularly regarding those aspects where disorder plays a prominent role such as are most transport phenomena. Amongst those, the behavior of the temperature dependence of the thermal conductivity $\kappa(T)$ of glasses and disordered crystals continues to attract a significant research effort. ${ }^{1}$ The most intriguing manifestation of the scattering of heat waves by either topological, ${ }^{1}$ orientational, ${ }^{2}$ chemical disorder, ${ }^{3}$ defect crystals, ${ }^{4}$ or even crystals with minimum disorder ${ }^{5}$ concerns the quantitative similitude of $\kappa(T)$ within a relatively wide range of temperatures $(0.1-10 \mathrm{~K})$, independent of the chemical composition. In stark contrast, most of our understanding of such phenomena relies on phenomenological constructs such as the two-level systems ${ }^{1}$ (TLSs) or soft-potential ${ }^{6}$ models (SPMs). Within the microscopic realm, studies aiming to identify the entities able to scatter heat-carrying phonons are mostly carried out by computer simulations. ${ }^{7}$

Here, we report on how minor chemical details such as the change in position of a functional group, namely, the $\mathrm{OH}$ group of alcohols, within the same molecular skeleton, lead to significant changes in thermal transport properties. More specifically, below we describe the measurements of $\kappa(T)$ for crystalline and glassy forms of the two chemical isomers of propyl alcohol $\left(\mathrm{CH}_{3} \mathrm{CH}_{2} \mathrm{CH}_{2} \mathrm{OH}\right.$ and $\mathrm{CH}_{3} \mathrm{CHOHCH}_{3}$ referred to as 1-Pr and 2-Pr hereafter) and how such a relatively minor chemical change leads to rather different behaviors for this transport property. The interest in such an exercise stems from the fact that such a chemical difference which does not translate into significant changes in molecular volumes or dipole moments but rather on a change of molecular shape leads to astonishing disparate properties such as the melting $\left(T_{m}=148\right.$ and $\left.185 \mathrm{~K}\right)$, boiling $\left(T_{b}\right.$ $=370.4$ and $355.4 \mathrm{~K})$ and glass-transition $\left(T_{g}=98\right.$ and $115 \mathrm{~K}$ for 1-Pr and 2-Pr, respectively) temperatures. ${ }^{8}$ Even more strikingly, the crystalline ground states of both materials also show marked differences in their crystal symmetry $\left(P 2_{1} / \mathrm{m}\right.$ and $P \overline{1}$, respectively) and cell dimensions (cell volumes of $V=604.91 \AA^{3}$ and $V=440.43 \AA^{3}$ for 1-Pr and 2-Pr, respectively ${ }^{9}$ ) as well as in their crystal packing densities, which differ by some $8 \%$, a value far in excess of that found in liquids. In addition, thermodynamic data such as the enthalpies of fusion yield values of $\Delta H_{f}=5.4 \mathrm{~kJ} \mathrm{~mol}^{-1}$ and $\Delta H_{f}=6.4 \mathrm{~kJ} \mathrm{~mol}^{-1},{ }^{10}$ which correlate with the difference of $37 \mathrm{~K}$ in the melting temperatures. The figures just quoted also provide a vivid reminder of the reasons behind the rather disparate crystallization kinetics of both isomers.

The topic of the chemical isomeric dependence of the thermodynamic functions of disordered matter has received attention ${ }^{11}$ in a recent study which shows that while, as expected, $T_{g}$ and $T_{b}$ show, in general, positive correlation, such temperatures are negatively correlated for isomeric liquids. Such an empirical finding, which is also borne out by the present material, has been rationalized ${ }^{11}$ in terms of entropy effects dominating over molecular interactions. Support for such a suggestion stems from the significant excess in specific heat of glassy 2-Pr with respect to $1-\mathrm{Pr},{ }^{12}$ which again correlates with the strong differences in the spectral frequency distributions of both isomers below $25 \mathrm{meV}$ as determined by neutron scattering. ${ }^{13}$

Our aim here is, thus, to identify the main microscopic entities giving rise to such disparate structural and thermodynamic behaviors by means of a study on the temperature dependence of a property such as the thermal conductivity, known since long ${ }^{14}$ to be sensitive to fine details concerning molecular motions. 


\section{EXPERIMENT}

The measurements for all the solid phases were carried out under equilibrium vapor pressure at $2-160 \mathrm{~K}$ by the steady-state potentiometric method using a special setup. ${ }^{15}$ The sample container is a stainless steel tube $40 \mathrm{~mm}$ long and $22 \mathrm{~mm}$ in diameter, with a wall thickness of $0.3 \mathrm{~mm}$. The bottom of the container was fixed to the cool zone of the cryostat that is connected to a helium bath. Two copper wires, $1 \mathrm{~mm}$ in diameter, pass through the container perpendicular to its axis, which permitted measurement of the average temperature along the isothermal plane running across the sample. The wires were $12.3 \mathrm{~mm}$ apart along the container axis. At the outer surface of the container, copper sockets were soldered to the wires to capsules of two temperature sensors. The upper sensor is a Cernox-SD resistance thermometer (Lake Shore Cryotronics, Inc.) measuring the temperature difference; the lower sensor is a TSU-2 resistance thermometer (VNIIFTRI) used to stabilize and control the temperature.

A thermometer was used to measure the temperature along the sample, with the heat flow on and off. The liquid sample was put into the container of the measuring cell under ${ }^{4} \mathrm{He}$ superlinear gas flow. The container once filled with the sample was vacuum-tight covered with a copper cap and an indium ring. The air was pumped out from the container through a stainless steel capillary attached to the cap. Afterwards, helium gas was fed into the container. A heater was mounted on the cap, allowing to generate a heat flow over the sample.

The statistical error in the thermal conductivity coefficient was below $3 \%$ for the whole range of measuring temperatures. The total measurement error $(\sim 10 \%)$ was mainly connected with the systematic error in the measurement of geometrical parameters (e.g., inner container cross section and spacing between thermometers).

Materials with a stated purity of 99.9\% (Chromasolv, Sigma-Aldrich) were used. The preparation of the glass and fully ordered monoclinic phase was carried out following previously described procedures. ${ }^{10}$ The different phases were prepared within the container using the same liquid sample. In short, the glasses were prepared by very fast cooling (above $50 \mathrm{~K} \mathrm{~min}^{-1}$ ) of the liquids through their glasstransition regions. Crystallization of the supercooled liquids into the orientationally ordered crystals is easily achieved for 2-Pr, but takes several hours at temperatures about $135 \mathrm{~K}$ for 1-Pr. Good quality crystals were grown by annealing for two days at temperatures close to $T_{m}$.

Some preliminary data concerning 1-Pr within its supercooled liquid range have already been reported within a study centered on a different topic. ${ }^{16}$

\section{RESULTS}

Figure 1 depicts the temperature dependence of both glassy and crystalline forms of isomeric propanols. The strikingly mild temperature dependence of data concerning glassy 1-Pr contrasts with its strong increase at low temperatures. Also, data for glassy 2-Pr come to be nearly superposable to

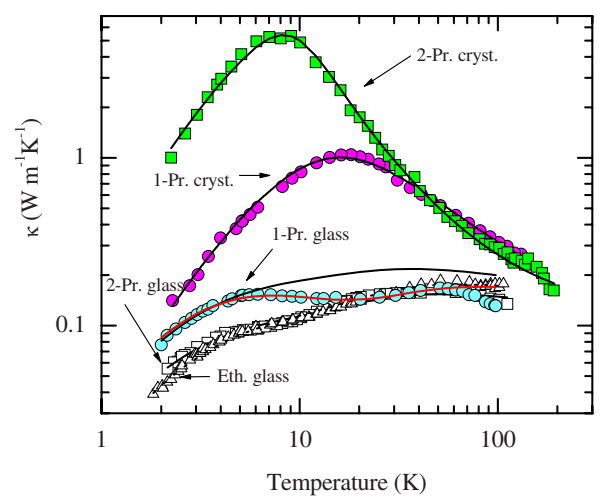

FIG. 1. (Color online) The thermal conductivity of both isomeric alcohols as compared to that measured for glassy ethanol (Ref. 2). Experimental data are depicted by symbols: open triangles for glassy ethanol, open squares for 2-propanol, and filled cyan circles for glassy 1-propanol. Filled purple circles depict data for the orientationally ordered crystals of 1-propanol and filled green squares show data for crystalline 2-propanol. Solid black lines are fitted curves using the soft potential model and yield parameter values shown in Table I. The solid red line corresponds to the calculation including the resonant-scattering term given by Eq. (9).

those measured for glassy $\mathrm{EtOH}^{2}$ At any rate, the most intriguing result concerns the significantly larger values of $\kappa(T)$ for glassy 1-Pr with respect to 2-Pr since, on simple grounds, one might expect that the phonon-gas model relating the conductivity with the phonon specific heat $C_{\mathrm{ph}}(T)$, sound velocity $c_{s}$, and phonon mean free path $l$, that is,

$$
\kappa(T)=\frac{1}{3} C_{\mathrm{ph}} c_{s} l,
$$

is expected to hold. Since the total specific heat of glassy 2-Pr exceeds that of 1-Pr at low and moderately low temperatures (i.e., below some $40 \mathrm{~K}),{ }^{10,12}$ and, on the other hand, there seems to be no large differences in the elastic behavior of both glasses, ${ }^{17}$ one would then expect the conductivity of glassy 2-Pr ought to exceed that of 1-Pr at least below $40 \mathrm{~K}$, a fact not borne out by experiment.

The temperature dependence of the thermal conductivity for a dielectric molecular crystal is known to be aptly described in terms of the Debye-Peierls model for an isotropic solid, where differences between acoustic phonons having different polarizations are disregarded. In short, this quantity is expressed in terms of an effective relaxation time $\tau(x)$ for phonon scattering as ${ }^{18,19}$

$$
\kappa(T)=\frac{k_{B}^{4} T^{3}}{2 \pi^{2} \hbar^{3} c_{s}} \int_{0}^{\Theta / T} d x \tau(x) \frac{x^{4} e^{x}}{\left(1-e^{x}\right)^{2}},
$$

where $x=\hbar \omega / k_{B} T, c_{s}$ is the speed of sound averaged over longitudinal and transverse polarizations, and $\Theta$ is the Debye temperature. The inverse relaxation rate $\tau^{-1}(\omega)$ is assumed to obey the Matthiesen rule and, therefore, can be expressed as a sum of rates representing different processes leading to phonon scattering. For an ordered crystal, ${ }^{20}$ it is expected that the dominant mechanisms able to scatter heat-carrying 
TABLE I. Parameter values entering the calculation of $\kappa(T)$ for the two crystalline solids. The value for the Debye temperature $\Theta$ was chosen such as to give an adequate description of $\kappa(T)$ in the high-temperature region. Data for grain boundary scattering yield a lower bound for the crystallite size $L>10^{-3} \mathrm{~m}$.

\begin{tabular}{lcccccc}
\hline \hline Sample & $\begin{array}{c}c_{s} \\
\left(\mathrm{~m} \mathrm{~s}^{-1}\right)\end{array}$ & $\begin{array}{c}B \\
\left(\times 10^{-18} \mathrm{~s} \mathrm{~K}^{-1}\right)\end{array}$ & $\begin{array}{c}E_{U} \\
(\mathrm{~K})\end{array}$ & $\begin{array}{c}D_{\mathrm{dis}} \\
\left(\times 10^{5}\right)\end{array}$ & $\begin{array}{c}C_{R} \\
\left(\times 10^{45} \mathrm{~s}^{3}\right)\end{array}$ & $\begin{array}{c}\Theta \\
(\mathrm{K})\end{array}$ \\
\hline Crystal EtOH & 1600 & 7 & 42 & 11 & 1 & 155 \\
Crystal 1-Pr & 1888 & 4.1 & 50 & 21 & 6.5 & 140 \\
Crystal 2-Pr & 1732 & 7.6 & 46.5 & 2.3 & 1.1 & 140 \\
\hline \hline
\end{tabular}

phonons will concern anharmonic umklapp processes with a rate $\tau_{U}^{-1}$, Casimir scattering from grain boundaries given by $\tau_{B}^{-1}$, and scattering by dislocations $\tau_{\text {dis }}^{-1}$ and Rayleigh scattering from point defects $\tau_{R}^{-1}$. The total rate is, thus,

$$
\tau^{-1}(\omega, T)=\tau_{U}^{-1}(\omega, T)+\tau_{B}^{-1}(\omega, T)+\tau_{\text {dis }}^{-1}(\omega, T)+\tau_{R}^{-1}(\omega, T)
$$

and relevant expressions for all the scattering processes are given by

$$
\begin{gathered}
\tau_{U}^{-1}(\omega, T)=B \omega^{2} T \exp \left(-E_{U} / T\right), \\
\tau_{B}^{-1}(\omega, T)=c_{s} / L, \\
\tau_{d i s}^{-1}(\omega, T)=D_{d i s} \omega, \\
\tau_{R}^{-1}(\omega, T)=C_{R} \omega^{4} .
\end{gathered}
$$

The relevant parameters entering the above expressions are the frequency factor $B$ and activation energy $E_{U}$ for the $U$ processes, the crystallite size $L$, the dislocation scattering strength $D_{\text {dis }}$, and the Rayleigh strength $C_{R}$. Equations (3) -(7) are able to describe the data for the ordered crystalline solids, and the numerical estimates for the best-fit parameters are collected in Table I and are compared to data for ordered crystalline ethanol, which constitutes a simpler system for which a lattice dynamic model has been reported ${ }^{21}$ and both its dynamics and thermodynamics of its known polymorphs have been studied extensively. ${ }^{22}$

The most remarkable differences in the parameter values given above concern the significantly larger values for those describing the strength of dislocations and Rayleigh scattering for 1-Pr if compared to the other two alcohols. In fact, the dislocation strength for crystalline 1-Pr comes to be close to 1 order of magnitude larger than that for 2-Pr, and the same applies to the Rayleigh term which shows a sixfold increase. The results, thus, depict crystalline 1-Pr as a highly defective solid where very strong scattering of heat-carrying phonons overcompensates the effects of a larger value for the sound velocity.

The temperature dependence of $\kappa(T)$ for both glasses was first analyzed on phenomenological grounds using the SPM, ${ }^{23-25}$ which portrays phonon scattering as mainly caused by low-energy excitations of a strongly anharmonic ensemble of particles. The scattering rate of acoustic phonons in a disordered system is given by the sum of three terms describing scattering by the tunnel states, classical re- laxors, and soft quasiharmonic vibrations, and reads

$$
\begin{aligned}
\tau_{G}^{-1}= & \bar{C} \pi \omega \tanh \left(\frac{\hbar \omega}{2 k_{B} T}\right)+\bar{C} \pi \omega\left[\frac{T}{W}\right]^{3 / 4} \ln ^{-1 / 4}\left(\frac{1}{\omega \tau_{0}}\right) \\
& +\frac{\bar{C} \pi \omega}{6 \sqrt{2}}\left[\frac{\hbar \omega}{W}\right]^{3} .
\end{aligned}
$$

Here, $\tau_{0}$ is the inverse of an attempt frequency and is of the order of $10^{-13} \mathrm{~s}$, which, when the sound wave frequencies $(10-100 \mathrm{GHz})$ are accounted for, yields a logarithmic factor of $\ln ^{-1 / 4}\left(1 / \omega \tau_{0}\right) \approx 0.7$. The most relevant parameters are the dimensionless $\bar{C}$ parameter and the characteristic energy $W$. Equation (8) was able to account for data concerning 2-Pr, but failed to reproduce the anomalously large plateau found for 1-Pr. In fact, as shown in Fig. 1, an attempt to fit the conductivity above some $6 \mathrm{~K}$ with the expression given above leads to a significant overestimation of such a quantity up to the highest temperatures. The presence of such an anomalously large plateau is suggestive of the action of strong phonon scattering by processes that become activated at temperatures above some $6 \mathrm{~K}$ or so. To account for such an observation, we have included an additional term representing resonant scattering from molecular modes having an average frequency $\omega_{0}{ }^{26}$ This additional term which represents resonant scattering of heat-carrying phonons by oscillators having an average frequency of $\omega_{0}$ reads

$$
\tau_{\text {res }}^{-1}(\omega, T)=\frac{F \omega^{2} T^{n}}{\left[1-\frac{\omega^{2}}{\omega_{0}^{2}}\right]^{2}+\gamma\left[\frac{\omega}{\omega_{0}^{2}}\right]^{4}}
$$

and the derived values for the three adjustable parameters were found to be $F=3.2 \times 10^{-17} \mathrm{~s} \mathrm{~K}^{-1}, n=1.56, \omega_{0}=25 \mathrm{~K}$ $(\approx 2.16 \mathrm{meV})$, and $\gamma=0.001$. A reasonable though not so good fit could be obtained assuming a fixed, linear dependence with temperature. Both cases, however, provide very close estimates for the resonance frequency.

The numerical values found for the parameters entering Eq. (8) are given in Table II. Within those, the Debye coefficient for the cubic term in the temperature dependence of the specific heat $C_{D}$ reflects the significantly larger value for 2-Pr than 1-Pr, and contrasts with the far milder differences found for the coefficients of the linear and fifth-order terms that correspond to TLS and quasilocalized vibrations. ${ }^{12}$ The parameter $W$ characterizes the crossover from a regime dominated by phonon scattering by low-energy excitations (tunneling states and classical relaxors) into another scatter- 
TABLE II. Parameter values entering the calculation of $\kappa(T)$ for all the glass phases.

\begin{tabular}{lccccccc}
\hline \hline Sample & $\begin{array}{c}\rho_{m} \\
\left(\mathrm{~kg} / \mathrm{m}^{-3}\right)\end{array}$ & $\begin{array}{c}C_{D} \\
\left(\mathrm{~mJ} \mathrm{~mol}^{-1} \mathrm{~K}^{-4}\right)\end{array}$ & $\begin{array}{c}c_{s} \\
\left(\mathrm{~ms}^{-1}\right)\end{array}$ & $\begin{array}{c}\Theta_{D} \\
(\mathrm{~K})\end{array}$ & $\begin{array}{c}\bar{C} \\
\left(\times 10^{4}\right)\end{array}$ & $\begin{array}{c}\mathrm{W}^{\mathrm{a}} \\
(\mathrm{K})\end{array}$ & $\mathrm{W}^{\mathrm{b}}$ \\
\hline Glassy EtOH & 958.2 & 1.55 & 1558 & 108 & 8.8 & 4 & 4.14 \\
Glassy 1-Pr & 804 & 1.77 & 1727 & 103 & 3.1 & 3.3 & 3.24 \\
Glassy 2-Pr & 785 & 2.54 & 1543 & 98 & 5.4 & 3 & 2.88 \\
\hline \hline
\end{tabular}

${ }^{\mathrm{a}}$ This work.

${ }^{b}$ Values derived from analysis of the specific heat. Notice that the Debye temperatures are about one-half of those reported in Ref. 12 since a different definition is used there.

ing by soft quasiharmonic vibrations. It is related to the temperature of the maximum $T_{\max }$ of $\kappa(T) / T$ so that $W$ $\simeq 1.6 T_{\max }$. The values given in the table are close to the temperatures signaling the beginning of the plateau in $\kappa(T)$. The fact that two different experiments carried out for two different thermal properties such as the specific heat and $\kappa(T)$ yielded remarkably similar values constitutes a consistency check for both sets of measurements.

From the measured values of $\kappa(T)$ as well as from the phonon specific heat, estimates for the temperature dependence of the phonon mean free paths have been derived and the results are shown in Fig. 2. Data for glassy 1-Pr obviously show larger values for the mean free path than that for the other two glasses. The temperature dependence of $l$ shows three well differentiated regions, varying as $T^{-2}$ below $3 \mathrm{~K}$, a $T^{-3}$ regime within $3-10 \mathrm{~K}$, and, finally, a hightemperature region for $T>40 \mathrm{~K}$ where it goes as $T^{-1 / 2}$. The crossover points (i.e., the intercepts between double logarithmic lines) are about 4 and $20 \mathrm{~K}$, respectively. The former temperature comes close to the value found for the crossover parameter $W$, whereas the latter approaches the value given by the resonance frequency $\omega_{0}$. Finally, data for the coupling strength parameter $\bar{C}$ for both glasses give us a clue to understand the excess in conductivity of 1-Pr over 2-Pr at low temperatures. In other words, such a result tells that for temperatures below the plateau $(T<\approx 4 \mathrm{~K})$, scattering from low-energy excitations is stronger in 2-Pr than in 1-Pr.

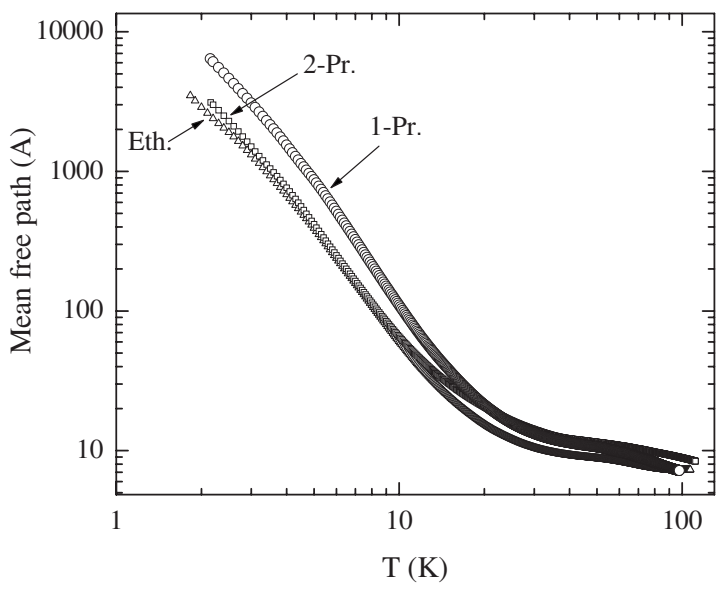

FIG. 2. Estimated phonon mean free paths for the three glasses here considered.

\section{DISCUSSION}

\section{A. Crystals}

The dependence of $\kappa(T)$ at low temperature for both 1-Pr and 2-Pr crystals follows a $T^{2}$ dependence rather than the $T^{3}$ behavior expected for monatomic crystals. ${ }^{20}$ The reasons behind such an apparent anomaly can be ascribed to interactions between acoustic phonons and the librations of orientationally ordered molecules as observed for methane crystals. ${ }^{27}$ Somewhat similar phenomena have been reported for clathrates, ${ }^{28}$ where the remarkable linear or $\kappa(T) \propto T^{3 / 2}$ dependences at low temperatures are understood as a consequence of the coupling of rattling motions of off-center atoms to phonons. ${ }^{3,29}$ Also, phenomenological treatments considering strain fields caused by wedge disclination dipoles ${ }^{4}$ lead to a clear crossover from $T^{3}$ to $T^{2}$ in the thermal conductivity at low temperature.

The specific heat data for both crystals ${ }^{10}$ follow a Debye, $C_{p} \propto T^{3}$, behavior up to about $3 \mathrm{~K}$ only. The ratio of the two quoted values for the Debye term $C_{D}(1-\operatorname{Pr}) / C_{D}(2-\operatorname{Pr})$ is about 0.71 , and is significantly smaller than that expected on the grounds of the density difference (i.e., $C_{p} \propto \rho^{-1}$ ), that is, 0.92 . The difference in sound velocities for the two crystals as given in Table I helps to solve such difficulty, since it provides an additional ratio of 0.92 to explain the difference in the low-temperature specific heat.

The maxima of $C_{p} / T^{3}$ curves are at $10.6 \mathrm{~K}$ for $1-\operatorname{Pr}$ and $8.6 \mathrm{~K}$ for 2-Pr and are accounted for by differences in the $Z(\omega)$ spectral frequency distributions for the two crystals reported in Ref. 10. The temperatures where the maxima in $C_{p} / T^{3}$ are located are, thus, close to the maxima of $\kappa(T)$ for the two crystals as shown in Fig. 1.

\section{B. Glasses}

The most striking difference between the measured $\kappa(T)$ for both glassy isomers concerns the substantially larger conductivity for 1-Pr with respect to 2-Pr below $15 \mathrm{~K}$ as well as the extremely large plateau exhibited by the former. A more detailed comparison of data is provided by plots shown in Fig. 3. There it is shown that at low temperatures, that is, below $3 \mathrm{~K}$, data for 1-Pr follow a power law $\kappa(T) \propto T^{m}$, with $m=0.94$. Within $3-5 \mathrm{~K}$, such a dependence gets smoother with $m=0.4$ and, finally, within 6-60 K, a remarkably mild dependence with temperature is found. The strong drop in $\kappa(T)$ apparent for $T>60 \mathrm{~K}$ is here ascribed to the onset of 




FIG. 3. (Color online) A comparison of the temperature dependence of the conductivites for both glasses. Circles depict data for 1-Pr and open squares data for 2-Pr. Lines drawn through data represent power laws which yield an approximate description of the temperature dependence of $\kappa(T)$ over restricted ranges of temperatures. The solid and dotted lines display the predictions grounded on the minimal thermal conductivity model (see text), which are calculated assuming that the only relevant dependence with temperature comes through the specific heat term.

low frequency motions which lead to the melting of the glass at $T_{g}=98 \mathrm{~K}$. The assignment is made on the grounds of previous muon spin relaxation results, ${ }^{30}$ which monitored the evolution with temperature of such motions by means of measurements of relaxation rates under a weak transverse field. Such experiments prove the dynamics within the micro- to nanoseconds time scales and, therefore, they have a direct bearing on heat transport processes. Results for 2-Pr show a softer temperature dependence for $T<3.5 \mathrm{~K}$ with $m=0.7$, followed by a region with $m=0.35$, which extends from 5 to $25 \mathrm{~K}$. Also, a basically temperature-independent region is found for $25-60 \mathrm{~K}$ and, finally, a strong drop of $\kappa(T)$ is again found for temperatures approaching the glass $\rightarrow$ liquid transition.

The results for $\kappa(T)$ commented on the above paragraph contrast with data for the specific heat, since all the coefficients entering the expansion with temperature of the specific heat are larger for 2-Pr than for 1-Pr. The results are, however, rationalized if consideration is made of the remarkable differences in the experimental vibrational frequency distributions at low frequencies of the two isomers as reported in Ref. 13. Figure 4 shows the strikingly large differences between the two spectral distributions. In particular, the excess modes, that is, the states giving rise to a frequency dependence stronger than $Z(\omega) \propto \omega^{2}$ observed for the crystals, are far more prominent in 2-Pr than in 1-Pr. Such states give rise to Boson peaks at about 2.5 and $1.8 \mathrm{meV}$ for $1-\mathrm{Pr}$ and 2-Pr, respectively, as shown in the inset of Fig. 4. The difference in the spectra at frequencies below some $5 \mathrm{meV}$ translates into rather different values for the $\bar{C}$ parameter which offset the differences in specific heat and, on such grounds, one expects from Eq. (2) to result in lower values for the conductivity of 2-Pr at low temperatures than those for 1-Pr.

Let us now consider the heat conductivity of both glasses for temperatures well above the plateau, that is, beyond some

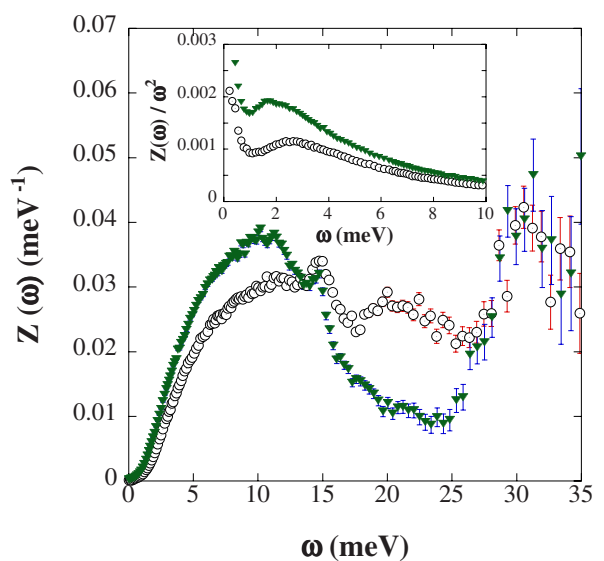

FIG. 4. (Color online) A comparison of the spectral frequency distributions for both glasses as derived from inelastic neutron scattering experiments reported in Ref. 13. Data for 1-Pr are depicted by open circles with a dot. Data for 2-Pr are shown by inverted triangles. The inset depicts the angle-averaged spectra $S(\omega)$ $=Z(\omega) / \omega^{2}$.

$5 \mathrm{~K}$, in some detail. To start with, we follow the same route as adopted for the analysis of the conductivity of several glasses, ${ }^{31}$ which is grounded on the concept of minimal thermal conductivity, which is reached when the distance between collisions of the phonon gas, that is, the $l$ parameter entering Eq. (1), equals the wavelength of the Debye elastic waves. Under such circumstances, heat is propagated by means of diffusionlike processes akin to those operative within the liquids. ${ }^{16}$ Also, following the suggestion made on Ref. 31, we take the high-temperature limit of such wavelength as twice the average intermolecular spacing, and we equate the latter to the distance $d=2 \pi / Q_{\max }$ given in terms of the position in momentum transfers of maxima of the static $S(Q)$ structure factor for both glasses, which become $Q_{p}$ $=1.56$ and $1.52 \AA^{-1}$, respectively, ${ }^{9}$ leading to estimates $l$ $=4.02$ and $4.13 \AA$ for 1 - $P r$ and 2-Pr, respectively. Taking, thus, the value of the high-temperature limit of the thermal conductivity within the glass state, that is, about $60 \mathrm{~J} \mathrm{~K}^{-1} \mathrm{~mol}^{-1}$ for both glasses, ${ }^{10}$ as well as the estimate of the sound velocities taken from the light-scattering study, ${ }^{17}$ we get estimates for the high-temperature limiting value of $\kappa(T)=0.21 \mathrm{~W} \mathrm{~m}^{-1} \mathrm{~K}^{-1}$ for both glasses which predict the correct order of magnitude. The result is, however, of limited validity since if we further assume as done in Ref. 31 that the temperature dependence comes from that of the specific heat only, the calculated thermal conductivities as shown in Fig. 3 fall with temperature far more rapidly than experiment.

Microscopically, the main difference between the two isomers concerns the two methyl groups present in 2-Pr versus only one for 1-Pr. Such chemical side groups are known to give rise to localized modes and in fact, are the main contributors to the strong features centered at $\omega=31 \mathrm{meV}$ in Fig. 4. Their effect on the thermal conductivity is, however, far more complex as proven by recent results on methyl-doped silica glass, ${ }^{32}$ which show that the conductivity at low temperature decreases with increasing methyl doping via a reduction of the participation ratio, that is, in the number of 
atoms which participate in a heat-carrying vibrational mode. The closeness of data for $\kappa(T)$ for 2-Pr and EtOH also points toward the effects of an additional methyl group in 2-Pr with respect to EtOH to scatter acoustic phonons. In fact, as shown in Table II, the Debye coefficient for the specific heat for glassy EtOH is about $60 \%$ that of 2-Pr, while $\kappa(T)$ for the two samples is superposable within temperatures of $2-10 \mathrm{~K}$. On such grounds, one expects to find for 2-Pr a scattering mechanism able to lower the conductivity to the same values as shown for EtOH. In this respect, the closeness of the estimated mean free path for both $\mathrm{EtOH}$ and 2-Pr within $2-10 \mathrm{~K}$ shown in Fig. 2 merits to be remarked.

In spite of the large difference in behavior of $\kappa(T)$ between the two isomers, it needs to be taken into account that the ansatz of quantitative universality, as proposed by Pohl et al., ${ }^{1}$ in terms of the ratio of the wavelength of the acoustic wave $\lambda$ to its mean free path $l$,

$$
\frac{\lambda}{l}=\pi^{2} \bar{C},
$$

still holds. In fact, data from Table II yield figures for such a quantity of $\simeq 2.8 \times 10^{-3}$ and $\simeq 5.3 \times 10^{-3}$ for both glasses and, therefore, our results are in quantitative agreement with data reported in Ref. 1, thus lending further support to the claim of "universal" behavior there expounded.

The present results also come into line with detailed studies on the molecular dynamics of 1-Pr carried out by means of NMR relaxation spectrometry ${ }^{33}$ and quasielastic neutron scattering, ${ }^{34}$ which depict rather complex molecular motions. In fact, both studies report on a minimum of three motional processes observable by both techniques within the normal and deeply supercooled liquids, which render inadequate the description of the molecular motions in terms of that for a rigid body.

\section{Origin of the difference in thermal conductivity}

Our ansatz of ascribing the mesmerizingly large plateau of data for 1-Pr to resonant scattering of phonons comes into line with other efforts dealing with thermal conduction in defect crystals and glasses. ${ }^{4,35}$ The phenomenon is known since pioneering studies on alkali halides ${ }^{26}$ doped with molecular ions which are able to reorient within the crystalline matrix. Here, we pursue a similar approach to that employed for the analysis of the conductivity of glassy $\mathrm{Se}^{7}$ and assign the origin of the strong plateau to the combined effects of relaxational scattering as well as to scattering from the low frequency tail of the librational contribution to the total $Z(\omega)$. Additional support for such an assignment comes from detailed lattice and molecular dynamics on ordered-crystalline (FOC) and orientationally disordered (OG) ethanol, ${ }^{21}$ which served to set bounds beyond which all phonon eigenvectors include substantial molecular-deformational components. More specifically, comparison of experimental data for the $Z(\omega)$ of fully hydrogenated and fully deuterated ethanol shows that an isotopic effect could be followed down to $1.65 \mathrm{meV}(\approx 19 \mathrm{~K})$ and $0.5 \mathrm{meV}(\approx 6 \mathrm{~K})$ for FOC and OG samples, respectively. The finding is interpreted as a direct evidence of a substantial contribution of the molecular libra- tions to $Z(\omega)$. As far as the kind of internal molecular motions able to undergo a strong coupling to the acoustic phonons is concerned, the lowest lying vibrational motions involve the $\mathrm{C}-\mathrm{C}$ torsion. The characteristic energies involved in such motions correspond to transitions between molecular conformations and are estimated to be as low as $35 \mathrm{~K}$ with respect to the ground state ${ }^{36}$ for the free molecule. In contrast, the most relevant internal coordinate of 2-Pr involves rotation about the $\mathrm{C}-\mathrm{O}$ bond, for which the most recent estimate yields characteristic energies of about $120 \mathrm{~K},{ }^{37}$ which are well above the energies of transverse acoustic phonons. ${ }^{21}$

As mentioned in the Introduction, the main difference between 1-Pr and 2-Pr concerns their molecular shapes. The latter is a nearly prolate top with asymmetry parameter $\sigma$ $=-0.84$, whereas $2-\operatorname{Pr}$ is a nearly oblate top with $\sigma=0.72 .{ }^{36,37}$ Transitions between the possible conformers in 1-Pr connecting different molecular structures which differ by the value of the dihedral angle subtended by the C-C-C-O skeleton involve a significant change in molecular shape since the ground state is characterized by principal moments of inertia of $36.9,18.6$, and $45.4 \mathrm{amu} \AA^{2}$, respectively, whereas the molecular parameters for the fully extended molecular conformation are 125.2, 108.7, and $134.4 \mathrm{amu} \AA^{2}$. Notice that all possible transitions in 2-Pr which only involve reorientation of the $\mathrm{OH}$ group with respect to the molecular frame lead to minor variations in molecular shape and, indeed, the asymmetry parameter for this molecule can change by 0.06 unit at most. The spectrum of such transitions for the free (gas phase) molecules has recently been reanalyzed ${ }^{36,37}$ due to its interest in astrophysics. From the differences in energy between 1-Pr conformers there reported, it can be inferred that these kinds of molecular motions will certainly hybridize with the acoustic excitations, giving rise to a substantial density of states at a few meV frequencies, in much the same way as we found for glassy $\mathrm{Se}^{7}$ in an earlier study. On such grounds as well as the proximity of the resonance frequency $\omega_{0}=25 \mathrm{~K}$ to the free-molecule value, we expect that transitions within different conformers in 1-Pr will be coupled to the elastic fields, a phenomenon absent in 2-Pr due to both the small change in molecular shape and the relatively high energy of the lower-lying conformational transition. Such a view is also consistent with the significant difference in the elastic properties of both samples as revealed by optical spectroscopy. ${ }^{17}$ The results here reported, thus, fit within the scenario sketched a long time ago as a result of modeling work on alkali halides, ${ }^{35}$ where the presence of a plateau in the thermal conductivity was mostly ascribed to elastic effects involved in the reorientations of $\mathrm{CN}$ dipoles within a $\mathrm{KBr}$ matrix. Also, results for glassy $\mathrm{Se}$ where an extended plateau in $\kappa(T)$ was also observed were accounted for in terms of chain motions akin to those here described.

\section{CONCLUDING REMARKS}

The data reported here, while confirming the general validity of claims of universality for this transport property, remind us of the semiquantitative character of such universal behavior as shown by the rather drastic effects of a minor 
chemical detail. The ultimate reasons for such disparate behaviors has to be sought in specific details concerning the potential energy surfaces of both materials. The difference in potential energy landscapes reveals itself in the vast difference in crystallization kinetics of both materials, since the formation of crystallites within the melt involves an exhaustive exploration of the free energy landscape.

Our current results for the thermal conductivity of both glasses show that, apart from the remarkable effects of the action of an internal degree of freedom in 1-Pr, both conduc- tivities at temperatures above $25 \mathrm{~K}$ or so can be understood on semiquantitative grounds in terms of the minimal conductivity concept, where heat is transferred by both acoustic phonons and other diffusive modes. The latter will dominate the heat transfer processes at the higher temperatures where molecular reorientational motions will become thermally activated. Such conditions lead to strong phonon scattering by rotational excitations, which is known to be very weakly dependent on temperature. ${ }^{38}$ *jbermejo@we.lc.ehu.es

${ }^{1}$ R. O. Pohl, Xiao Liu, and E. Thompson, Rev. Mod. Phys. 74, 991 (2002).

${ }^{2}$ A. I. Krivchikov, A. N. Yushchenko, V. G. Manzhelii, O. A. Korolyuk, F. J. Bermejo, R. Fernandez-Perea, C. Cabrillo, and M. A. Gonzalez, Phys. Rev. B 74, 060201 (2006); E. R. Grannan, M. Randeria, and J. P. Sethna, ibid. 41, 7784 (1990).

${ }^{3}$ F. Bridges and L. Downward, Phys. Rev. B 70, 140201(R) (2004); K. A. Topp, E. J. Thompson, and R. O. Pohl, ibid. 60, 898 (1999).

${ }^{4}$ S. E. Krasavin and V. A. Osipov, J. Phys.: Condens. Matter 13, 1023 (2001).

${ }^{5}$ J. P. Wrubel, B. E. Hubbard, N. I. Agladze, A. J. Sievers, P. P. Fedorov, D. I. Klimenchenko, A. I. Ryskin, and J. A. Campbell, Phys. Rev. Lett. 96, 235503 (2006).

${ }^{6}$ V. G. Karpov, M. I. Klinger, and F. N. Ignatiev, Solid State Commun. 44, 333 (1982); U. Buchenau, Y. M. Galperin, V. L. Gurevich, D. A. Parshin, M. A. Ramos, and H. R. Schober, Phys. Rev. B 46, 2798 (1992).

${ }^{7}$ See, for instance, X. Yu and D. M. Leitner, Phys. Rev. B 74, 184305 (2006); F. J. Bermejo, E. Enciso, A. Criado, J. L. Martinez, and M. Garcia-Hernandez, ibid. 49, 8689 (1994).

${ }^{8}$ R. C. Whilhoit and B. J. Zwolinski, J. Phys. Chem. Ref. Data Suppl. 2, 74 (1973); 2, 82 (1973).

${ }^{9}$ C. Talon, F. J. Bermejo, C. Cabrillo, G. J. Cuello, M. A. Gonzalez, J. W. Richardson, Jr., A. Criado, M. A. Ramos, S. Vieira, F. L. Cumbrera, and L. M. Gonzalez, Phys. Rev. Lett. 88, 115506 (2002).

${ }^{10}$ G. J. Cuello, C. Talon, F. J. Bermejo, and C. Cabrillo, Appl. Phys. A: Mater. Sci. Process. 74, S552 (2002); C. Talon, M. A. Ramos, S. Vieira, I. Shmytko, N. Afonikova, A. Criado, G. Madariaga, and F. J. Bermejo, J. Non-Cryst. Solids 287, 226 (2001).

${ }^{11}$ Li-Min Wang and R. Richert, J. Phys. Chem. B 111, 3201 (2007).

${ }^{12}$ M. A. Ramos, C. Talon, and S. Vieira, J. Non-Cryst. Solids 307310, 80 (2002).

${ }^{13}$ C. Talon, G. J. Cuello, M. A. Gonzalez, F. J. Bermejo, C. Cabrillo, and R. Connatser, Chem. Phys. 292, 263 (2002).

${ }^{14}$ W. D. Seward and V. Narayanamurti, Phys. Rev. 148, 463 (1966); W. D. Seward, V. Narayanamurti, and R. O. Pohl, ibid. 148, 481 (1966); M. Wagner, ibid. 131, 1443 (1963).

${ }^{15}$ A. I. Krivchikov, B. Ya Gorodilov, O. A. Korolyuk, V. G. Manzhelii, H. Conrad, and W. Press, J. Low Temp. Phys. 139, 693 (2005); A. I. Krivchikov, V. G. Manzhelii, O. A. Korolyuk, B. Ya. Gorodilov, and O. O. Romantsova, Phys. Chem. Chem. Phys. 7, 728 (2005); A. I. Krivchikov, B. Ya. Gorodilov, and O.
A. Korolyuk, Instrum. Exp. Tech. 48, 417 (2005).

${ }^{16}$ A. I. Krivchikov, A. N. Yushenko, O. A. Korolyuk, F. J. Bermejo, C. Cabrillo, and M. A. Gonzalez, Phys. Rev. B 75, 214204 (2007).

${ }^{17}$ Y. Takagi, T. Yano, and S. Kojima, Jpn. J. Appl. Phys., Part 1 38, 3066 (1999).

${ }^{18}$ V. V. Sumarokov, P. Stachowiak, J. Mucha, and A. Jezowski, Phys. Rev. B 74, 224302 (2006).

${ }^{19}$ P. Stachowiak, V. V. Sumarokov, and A. Jezowski, Phys. Rev. B 69, 024305 (2004); P. Stachowiak, V. V. Sumarokov, J. Mucha, and A. Jezowski, ibid. 67, 172102 (2003).

${ }^{20}$ J. Callaway, Phys. Rev. 113, 1046 (1959); C. Herring, ibid. 95, 954 (1954).

${ }^{21}$ C. Talon, M. A. Ramos, S. Vieira, G. J. Cuello, F. J. Bermejo, A. Criado, M. L. Senent, S. M. Bennington, H. E. Fischer, and H. Schober, Phys. Rev. B 58, 745 (1998).

${ }^{22}$ M. A. Ramos, S. Vieira, F. J. Bermejo, J. Dawidowski, H. E. Fischer, H. Schober, M. A. Gonzalez, C. K. Loong, and D. L. Price, Phys. Rev. Lett. 78, 82 (1997); A. Criado, M. JimenezRuiz, C. Cabrillo, F. J. Bermejo, M. Grimsditch, H. E. Fischer, S. M. Bennington, and R. S. Eccleston, Phys. Rev. B 61, 8778 (2000); M. Jimenez-Ruiz, A. Criado, F. J. Bermejo, G. J. Cuello, F. R. Trouw, R. Fernandez-Perea, H. Löwen, C. Cabrillo, and H. E. Fischer, Phys. Rev. Lett. 83, 2757 (1999); C. Cabrillo, F. J. Bermejo, M. Jimenez-Ruiz, M. T. Fernandez-Diaz, M. A. Gonzalez, and D. Martin y Marero, Phys. Rev. B 64, 064206 (2001); H. E. Fischer, F. J. Bermejo, G. J. Cuello, M. T. Fernandez-Diaz, J. Dawidowski, M. A. Gonzalez, H. Schober, and M. Jimenez-Ruiz, Phys. Rev. Lett. 82, 1193 (1999); M. A. Miller, M. Jimenez-Ruiz, F. J. Bermejo, and N. O. Birge, Phys. Rev. B 57, R13977 (1998).

${ }^{23}$ U. Buchenau, Yu. M. Galperin, V. L. Gurevich, D. A. Parshin, M. A. Ramos, and H. R. Schober, Phys. Rev. B 46, 2798 (1992).

${ }^{24}$ M. A. Ramos and U. Buchenau, Phys. Rev. B 55, 5749 (1997).

${ }^{25}$ F. J. Bermejo, C. Cabrillo, M. A. Gonzalez, and M. L. Saboungi, J. Low Temp. Phys. 139, 567 (2005).

${ }^{26}$ R. O. Pohl, Phys. Rev. Lett. 8, 481 (1962); A. Kumar, A. K. Srivastava, and G. S. Varma, Phys. Rev. 178, 1480 (1969).

${ }^{27}$ P. Stachowiak, E. Pisarska, A. Jezowski, and A. I. Krivchikov, Phys. Rev. B 73, 134301 (2006); P. Stachowiak, E. Pisarska, A. Jezowski, and A. I. Krivchikov, Europhys. Lett. 74, 96 (2006).

${ }^{28}$ B. C. Sales, B. C. Chakoumakos, R. Jin, J. R. Thompson, and D. Mandrus, Phys. Rev. B 63, 245113 (2001); G. S. Nolas, T. J. R. Weakley, J. L. Cohn, and R. Sharma, ibid. 61, 3845 (2000); A. I. Krivchikov, B. Y. Gorodilov, O. A. Korolyuk, V. G. Manzhe- 
lii, O. O. Romantsova, H. Conrad, W. Press, J. S. Tse, and D. D. Klug, ibid. 73, 064203 (2006).

${ }^{29}$ J. S. Tse, D. D. Klug, J. Y. Zhao, W. Sturhahn, E. E. Alp, J. Baumert, C. Gutt, M. R. Johnson, and W. Press, Nat. Mater. 4, 917 (2005).

${ }^{30}$ F. J. Bermejo, I. Bustinduy, M. A. Gonzalez, S. H. Chong, C. Cabrillo, and S. F. J. Cox, Phys. Rev. B 70, 214202 (2002).

${ }^{31}$ D. G. Cahill and R. O. Pohl, Phys. Rev. B 35, 4067 (1987).

${ }^{32}$ X. Yu and D. M. Leitner, Phys. Rev. B 74, 184305 (2006).

${ }^{33}$ M. Pöschl and H. G. Hertz, J. Phys. Chem. 98, 8195 (1994).

${ }^{34}$ F. J. Bermejo, W. S. Howells, M. Jimenez-Ruiz, M. A. Gonzalez,
D. L. Price, M. L. Saboungi, and C. Cabrillo, Phys. Rev. B 69 , 174201 (2004).

${ }^{35}$ E. R. Grannan, M. Randeria, and J. P. Sethna, Phys. Rev. B 41, 7799 (1990).

${ }^{36}$ A. Maeda, F. C. De Lucia, E. Herbst, J. C. Pearson, J. Riccobono, E. Trosell, and R. K. Bohn, Astrophys. J., Suppl. Ser. 162,. 428 (2006).

${ }^{37}$ A. Maeda, I. R. Medvedev, F. C. De Lucia, and E. Herbst, Astrophys. J., Suppl. Ser. 166, 650 (2006).

${ }^{38}$ O. I. Pusky and V. A. Kosntantinov, Cent. Eur. J. Phys. 4, 210 (2006). 